\title{
Killer immunoglobulin-like receptors/human leukocyte antigen class-I, a crucial immune pathway in cancer
}

\author{
Yi Xu ${ }^{1,2}$, Lei Wang ${ }^{1}$, Wei $\mathrm{Li}^{1}$, Bin Chen ${ }^{1}$, Yu Liu ${ }^{1,2}$, Hao Wang ${ }^{1,2}$, Sha Zhao ${ }^{1}$, Lingyun Ye ${ }^{1}$, Yayi He ${ }^{1}$, \\ Caicun Zhou ${ }^{1}$
}

${ }^{1}$ Department of Medical Oncology, Shanghai Pulmonary Hospital, Tongji University Medical School Cancer Institute, Tongji University School of Medicine, Shanghai 200433, China; ${ }^{2}$ Tongji University, Shanghai 200433, China

Contributions: (I) Conception and design: Y He, C Zhou; (II) Administrative support: Y He, C Zhou; (III) Provision of study materials or patients: L Wang, W Li, B Chen; (IV) Collection and assembly of data: Y Liu, H Wang; (V) Data analysis and interpretation: S Zhao, L Ye; (VI) Manuscript writing: All authors; (VII) Final approval of manuscript: All authors.

Correspondence to: Yayi He. Department of Medical Oncology, Shanghai Pulmonary Hospital, Tongji University Medical School Cancer Institute, Tongji University School of Medicine, Shanghai 200433, China. Email: 2250601@qq.com.

\begin{abstract}
Natural killer cells (NK cells) play a crucial role in tumor immunity. The function of the NK cells is regulated by various receptors expressed on the surface. Among them, the killer immunoglobulin-like receptor (KIR) is one of the most important. The ligand of KIR is major histocompatibility complex class-I (MHC class-I), which is also called human leukocyte antigen class-I (HLA class-I). The combination of HLA class-I and inhibitory KIRs could inhibit NK cells and induce autoimmune tolerance. Inhibitory KIRs were highly expressed on malignant tumor patients, which were related to poor prognosis. KIR/HLA class-I pathway affected the clinical outcomes of cancer through several mechanisms, and inhibitory KIRs could be an ideal target of immunotherapy strategy.
\end{abstract}

Keywords: Killer immunoglobulin-like receptor (KIR); leukocyte antigen class-I (HLA class-I); immune therapy; cancer

Submitted Dec 03, 2019. Accepted for publication Dec 30, 2019.

doi: $10.21037 /$ atm.2020.01.84

View this article at: http://dx.doi.org/10.21037/atm.2020.01.84

\section{Introduction}

Cancer is a major disease that poses a serious threat to human health. There were 18.1 million new cases and 9.6 million cancer deaths in 2018, according to the data on 36 cancers spanning 185 countries conducted by the Global Cancer Observatory. It was lung cancer that ranked first in both morbidity and mortality (1). Another system analysis of 29 cancer groups worldwide showed that in the past 10 years, from 2006 to 2016, cancer cases have increased by 28\% (2).

The development of cancer is directly related to the host's immune function. Tumor cells with stronger immunogenicity can be recognized and eliminated by the immune system, while some others can escape from it through several mechanisms and then develop into cancers (3). Animal experiments demonstrated a bi-directional selection between tumor cells and the immune system. After killing more immunogenic tumor cells in immunocompetent mice, the immune system tends to select and keep less immunogenic cells that do not carry antigens. Moreover, the immune system also affects the tumor microenvironment, promoting, and providing the right conditions for tumor cells to grow $(4,5)$.

The innate immune response is the first line in tumor immune response, where natural killer cells (NK cells) play the most significant role. Most (about 90\%) human NK cells express CD56 in low-density and Fcy receptor III (FcyRIII, CD16) at a high level (6). No specific antigen recognition receptor exists on the surface of NK cells. The recognition and killing function of NK cells is regulated by the inhibitory and activating receptors expressed on their surface. 
Killer immunoglobulin-like receptor (KIR) is a superfamily of immunoglobulin existing right on the surface of NK cells, consisting of Ig-like domains that bind to HLA class-I molecules, which help them distinguish between "the self" and "the non-self." KIRs involve in education and selection during the process of NK cell maturation (7-9). This kind of interaction between HLA class-I molecules expressed on normal tissue cells, and inhibitory KIRs on the surface of NK cells shapes the body's autoimmune tolerance.

Cancer cells are characterized mostly by the loss or less presence of HLA class-I molecules that can be recognized as "non-self" by NK cells, which facilitates the tumor immune response (10). In the growth progress of tumors, that those tumor cells expressing HLA class-I molecules as normal tissues fail to activate the killing function of NK cells, which paves the way for immune escape and further development of tumor (4). What revealed by a large quantity of evidence is that inhibitory KIRs were highly expressed on the NK cells and tumor infiltration lymphocytes (TILs) in malignant tumor patients, and the loss or lower expression of inhibitory KIRs was correlated with better clinical outcomes and therapy response (11-13). Therefore, to blockade the inhibitory KIRs might be a promising immunotherapy strategy to activate and enhance immune response and reverse tumor immune escape.

\section{KIRs are the key regulators of human NK cell function}

NK cell plays a crucial role as the first line in antitumor immunity, accounting for $10 \%$ to $20 \%$ of the total peripheral blood lymphocyte (8). KIRs belong to the immunoglobulin superfamily, which is coded by 14 polymorphic genes (KIR2DL1-5, KIR3DL1-3, KIR2DS1-5, KIR3DS1) and structured by extracellular domains and intracytoplasmic tail $(11,14)$. There exist 2 or 3 Ig-like domains that bind to HLA class-I molecules on the extracellular domains classifying KIR into KIR2D and KIR3D. KIR2DL and LIR3DL are those contain long (L) intracytoplasmic tail characterized by immune receptor tyrosine - based inhibitory motifs (ITIMs) to transduce inhibitory signals to restrain the activity of NK cells, while most KIR2DS and KIR3DS work as activating receptors with the lack of ITIMs on the short (S) intracytoplasmic tail (15).

According to existing literature, the function of NK cells is regulated by various receptors expressed on the surface, including specific natural killing receptors (NKRs), cytokine receptors and KIRs, etc., among which inhibitory KIRs serve as the critical regulation $(8,16)$. Different inhibitory KIRs bind to different HLA subtypes, KIR2DL1 to HLACw2, -4, -5, -6, and KIR2DL2-3 to HLA-Cw1, -3, -7, and -8. Inhibitory KIRs recognize all HLA-C (14). KIRs are highly polymorphic molecules due to the diversity of KIR genes on the amount and allele, as well as KIR ligands HLA class-I molecules. In addition to the random expression of KIRs, the possible KIR-HLA combinations are of great diversity, which leads to the high plasticity of NK cells $(17,18)$.

\section{The HLA class-I plays a crucial role in tumor immune response and escape}

The HLA class-I molecules have been confirmed already the ligands of KIRs (12). The molecules are universally expressed on normal cells, involving in presenting antigen peptides towards specific T-cells and shaping autoimmune tolerance as well. Among them, the most significant one is HLA-C binding to inhibitory $\operatorname{KIR} 2 \mathrm{D}(8,17)$. In physiological situations, inhibitory KIRs bind to HLA class-I and take the dominant position, so that normal cells cannot be killed by NK cells. Cancer cells are characterized by the loss or less expression of HLA class-I molecules. That KIR-ligand mismatch transduces activating signals, lowers the sensitivity to inhibitory signals, and activates NK cells (10). Nevertheless, under the pressure of anti-tumor immunity, the expression of specific antigens decreases on some tumor cells, but that keeps unchanged in HLA class-I molecules, which unable to activate NK cells, then, this group of tumor cells escapes from immune clearance (4).

\section{KIR/HLA class-I has an association with clinical outcomes}

Increasing evidence showed that KIR/HLA class-I was correlated to the susceptibility and development of malignant tumors (including blood cancer), the loss or lower expression of inhibitory KIRs and HLA class-I molecules was related to better therapy response and clinical outcomes (11-13). In an analysis of over one hundred tumors decreases, the frequency of HLA class-I expression deletion and (or) reduction was from $16 \%$ to $80 \%$, highest in bladder, breast and prostate cancer, while lowest in renal cell carcinoma (RCC) and melanoma (19). Lower incidence of HLA class-I defect can be seen in leukemia and 
lymphoma, which probably results from the low sensitivity of tumor cells with high expression of HLA class-I to NK cell-mediated cytolysis (20). It was shown that inhibitory KIR-HLA mismatch was the only crucial prognostic factor in a prospective study enrolling sixteen consecutive patients with lymphoma or solid tumor (21). A study conducted on 62 lung cancer patients demonstrated KIR2D (L1, L3, L4, S4) and KIR3DL1 were expressed on both cancer cells and TILs. The KIR2D high expression on cancer cells is related to the high expression of KIR3DL1 on cancer cells and KIR2D, KIR3DL1 on TILs. There existed a correlation between the high expression of KIR3DL1 on cancer cells and that on TILs, as well as KIR2D on TILs and KIR3DL1 on both cells (11). Analysis on the KIR gene type found that KIR2DL4 and KIR3DL2-3 are present in all colorectal cancer patients and KIR3DL1 in over 96\%. KIRs also make difference in the relapse pf colorectal cancer. In relapse groups with locally advanced or metastatic colorectal cancer, KIR2DL1 was often observed but activated combination KIR2DS2-C1 was less. In the meanwhile, more frequent KIR2DL2, KIR2DS2-3 was observed in non-relapse groups (22). Among patients with leukemia, higher affinity was observed between KIR2DL2/KIR2DL3 and heterozygous HLA-C1 (8). That the significantly clinical decrease of acute myeloid leukemia (AML) relapse risk after transplanting hematopoietic stem cells with it proved the anti-leukemia function of KIR2DL5A (23). Activated KIR2D was also reported to be a protective factor. Activated KIR3DS1-2 was found protective of Hodgkin's lymphoma during its development, which is more notable in EpsteinBarr virus (EBV) positive patients (8). The same effect of activated KIR2DS3 was reported in acute lymphoblastic leukemia (ALL), AML, and chronic myeloid leukemia (CML), and the incidence was more frequent in leukemia with them combining (23).

As was proved, KIR-HLA class-I affected the antitumor therapy response. Besides, KIR-HLA mismatch (the absence of inhibitory KIR2DL5A, 2DL5B, 2DL5 genes) was correlated with better therapeutic efficacy in CML patients (24), the outcomes of leukemia patients taking hematopoietic stem cell transplantation (HSCT) could be notably improved. Evidence also showed that the KIRHLA mismatch resulted in a better prognosis for patients undergoing immune therapy involving NK cell function, for example, an anti-GD2 monoclonal antibody (mAb) with the ability to interfere with the HLA-C expression on primary tumor cells $(12,14)$.

Cytokines were found another approach to immune function regulation. An in vitro study revealed that inhibitory HLA/KIR blockade inducted NK cells to secrete IFN- $\alpha$ and IFN- $\gamma$. Inhibitory HLA/KIR blockade led to the increased expression of TNF in AML. Anti-inhibitory KIR drugs raised the expression of IFN- $\alpha$, TNF- $\alpha$, IL- 6 , and many other cytokines $(14,25)$.

\section{The expression of KIRs is associated with PD-1}

A critical pathway of tumor immune escape is programmed death-1 (PD-1) and programmed death ligand-1 (PD-L1), which can transduce inhibitory signals towards T-cells to restrain the killing activity of CTLs so that there exists a correlation between high expression of PD-1/PD-L1 and poor diagnosis of cancer (26). As is explained, high expression of inhibitory KIRs is associated with poor prognosis of patients with many malignant tumors. The latest study on 130 newly diagnosed non-small cell lung cancer (NSCLC) patients reported that PD-1 high expression was associated with the high expression of inhibitory KIR2DL1, 2DL3, 2DL4, 2DS4 on tumor cells and TILs. Undergoing anti-PD-1 monoclonal antibodies therapy, nearly half of NSCLC patients expressed inhibitory KIRs on tumor cells. The mechanism of this situation was the inhibitory HLA/KIR combined with a PD-1/PD-L1 signaling pathway, negatively adjusted tumor immunity, and facilitated immune escape (27).

\section{Summary}

There has been a growing recognition that surveillance is merely one aspect of the complicated relationship between the immune system and cancer. On the one hand, those tumor cells with stronger immunogenicity can be recognized and eliminated or inhibited by the immune system. On the other hand, tumor cells expressing HLA class-I normally will adapt to the immune system and keep growing. It has been proved that there exists a mutual selection between cancer and the immune system.

KIRs, including inhibitory KIRs and activating KIRs, play a key role in regulating the function of NK cells. The ligand of KIR is MHC class-I (or called HLA class-I). Autoimmune tolerance is shaped by the interaction between those HLA class-I molecules expressed on normal tissue cells and inhibitory KIRs on the surface of NK cells. The lower expression of HLA class-I on tumor cells can disable the inhibitory KIRs and activate the killing function of NK cells to eliminate them. KIR gene defect and KIR/HLA 
class-I mismatch is correlated with better therapy response and clinical outcomes. A correlation exists between KIR and PD-1. In this knowledge, we can predict KIR and KIR/HLA class-I pathway's new prognostic indexes and promisingly therapeutic target to cancers.

\section{Acknowledgments}

Funding: This study was supported in part by a grant from National Natural Science Foundation of China (81802255), Shanghai Pujiang Program (17PJD036) and a grant from Shanghai Municipal Commission of Health and Family Planning Program (20174Y0131), National Key Research \& Development Project (2016YFC0902300), Major disease clinical skills enhancement program of three-year action plan for promoting clinical skills and clinical innovation in municipal hospitals, Shanghai Shen Kang Hospital Development Center Clinical Research Plan of SHDC (16CR1001A), "Dream Tutor" Outstanding Young Talents Program (fkyq1901), key disciplines of Shanghai Pulmonary Hospital (2017ZZ02012), grant of Shanghai Science and Technology Commission (16JC1405900).

\section{Footnote}

Conflicts of Interest: The authors have no conflicts of interest to declare.

Ethical Statement: The authors are accountable for all aspects of the work in ensuring that questions related to the accuracy or integrity of any part of the work are appropriately investigated and resolved.

Open Access Statement: This is an Open Access article distributed in accordance with the Creative Commons Attribution-NonCommercial-NoDerivs 4.0 International License (CC BY-NC-ND 4.0), which permits the noncommercial replication and distribution of the article with the strict proviso that no changes or edits are made and the original work is properly cited (including links to both the formal publication through the relevant DOI and the license). See: https://creativecommons.org/licenses/by-nc-nd/4.0/.

\section{References}

1. Bray F, Ferlay J, Soerjomataram I, et al. Global cancer statistics 2018: GLOBOCAN estimates of incidence and mortality worldwide for 36 cancers in 185 countries. CA
Cancer J Clin 2018;68:394-424.

2. Global Burden of Disease Cancer Collaboration, Fitzmaurice C, Akinyemiju TF, et al. Global, Regional, and National Cancer Incidence, Mortality, Years of Life Lost, Years Lived With Disability, and Disability-Adjusted LifeYears for 29 Cancer Groups, 1990 to 2016: A Systematic Analysis for the Global Burden of Disease Study. JAMA Oncol 2018;4:1553-68.

3. Dunn GP, Old LJ, Schreiber RD. The immunobiology of cancer immunosurveillance and immunoediting. Immunity 2004;21:137-48.

4. Schreiber RD, Old LJ, Smyth MJ. Cancer immunoediting: integrating immunity's roles in cancer suppression and promotion. Science 2011;331:1565-70.

5. Aragon-Sanabria V, Kim GB, Dong C. From Cancer Immunoediting to New Strategies in Cancer Immunotherapy: The Roles of Immune Cells and Mechanics in Oncology. Adv Exp Med Biol 2018;1092:113-8.

6. Cooper MA, Fehniger TA, Caligiuri MA. The biology of human natural killer-cell subsets. Trends Immunol 2001;22:633-40.

7. Forlenza CJ, Boudreau JE, Zheng J, et al. KIR3DL1 Allelic Polymorphism and HLA-B Epitopes Modulate Response to Anti-GD2 Monoclonal Antibody in Patients With Neuroblastoma. J Clin Oncol 2016;34:2443-51.

8. Varbanova V, Naumova E, Mihaylova A. Killer-cell immunoglobulin-like receptor genes and ligands and their role in hematologic malignancies. Cancer Immunol Immunother 2016;65:427-40.

9. Kim S, Poursine-Laurent J, Truscott SM, et al. Licensing of natural killer cells by host major histocompatibility complex class-I molecules. Nature 2005;436:709-13.

10. Joncker NT, Raulet DH. Regulation of NK cell responsiveness to achieve self-tolerance and maximal responses to diseased target cells. Immunol Rev 2008;224:85-97.

11. He Y, Bunn PA, Zhou C, et al. KIR 2DL1, L3, L4, S4 and KIR 3DL1 protein expression in non-small cell lung cancer. Oncotarget 2016;7:82104-11.

12. Wang W, Erbe AK, Gallenberger M, et al. Killer immunoglobulin-like receptor (KIR) and KIR-ligand genotype do not correlate with clinical outcome of renal cell carcinoma patients receiving high-dose IL2. Cancer Immunol Immunother 2016;65:1523-32.

13. Venstrom JM, Zheng J, Noor N, et al. KIR and HLA genotypes are associated with disease progression and survival following autologous hematopoietic stem cell transplantation for high-risk neuroblastoma. Clin Cancer 
Res 2009;15:7330-4.

14. Romagné F, André P, Spee P, et al. Preclinical characterization of 1-7F9, a novel human anti-KIR receptor therapeutic antibody that augments natural killermediated killing of tumor cells. Blood 2009;114:2667-77.

15. Falco M, Moretta L, Moretta A, et al. KIR and KIR ligand polymorphism: a new area for clinical applications? Tissue Antigens 2013;82:363-73.

16. Dorak MT. Role of natural killer cells and killer immunoglobulin-like receptor polymorphisms: association of HLA and KIRs. Methods Mol Med 2007;134:123-44.

17. Leone $\mathrm{P}, \mathrm{De}$ Re V, Vacca A, et al. Cancer treatment and the KIR-HLA system: an overview. Clin Exp Med 2017;17:419-29.

18. Moretta A, Pende D, Locatelli F, et al. Activating and inhibitory killer immunoglobulin-like receptors (KIR) in haploidentical haemopoietic stem cell transplantation to cure high-risk leukaemias. Clin Exp Immunol 2009; 157:325-31.

19. Campoli M, Ferrone S. HLA antigen changes in malignant cells: epigenetic mechanisms and biologic significance. Oncogene 2008;27:5869-85.

20. Demanet C, Mulder A, Deneys V, et al. Downregulation of HLA-A and HLA-Bw6, but not HLA-Bw4, allospecificities in leukemic cells: an escape mechanism from CTL and NK attack? Blood 2004;103:3122-30.

21. Leung $W$, Handgretinger R, Iyengar R, et al. Inhibitory KIR-HLA receptor-ligand mismatch in autologous haematopoietic stem cell transplantation for solid tumour

Cite this article as: $\mathrm{Xu} \mathrm{Y,} \mathrm{Wang} \mathrm{L,} \mathrm{Li} \mathrm{W,} \mathrm{Chen} \mathrm{B,} \mathrm{Liu} \mathrm{Y,}$ Wang H, Zhao S, Ye L, He Y, Zhou C. Killer immunoglobulinlike receptors/human leukocyte antigen class-I, a crucial immune pathway in cancer. Ann Transl Med 2020;8(5):244. doi: 10.21037/atm.2020.01.84 and lymphoma. Br J Cancer 2007;97:539-42.

22. Beksac K, Beksac M, Dalva K, et al. Impact of "Killer Immunoglobulin-Like Receptor /Ligand" Genotypes on Outcome following Surgery among Patients with Colorectal Cancer: Activating KIRs Are Associated with Long-Term Disease Free Survival. PLoS One 2015;10:e0132526.

23. Stringaris K, Adams S, Uribe M, et al. Donor KIR Genes 2DL5A, 2DS1 and 3DS1 are associated with a reduced rate of leukemia relapse after HLA-identical sibling stem cell transplantation for acute myeloid leukemia but not other hematologic malignancies. Biol Blood Marrow Transplant 2010;16:1257-64.

24. Kreutzman A, Jaatinen T, Greco D, et al. Killer-cell immunoglobulin-like receptor gene profile predicts good molecular response to dasatinib therapy in chronic myeloid leukemia. Exp Hematol 2012;40:906-913.e1.

25. Vey N, Bourhis JH, Boissel N, et al. A phase 1 trial of the anti-inhibitory KIR mAb IPH2101 for AML in complete remission. Blood 2012;120:4317-23.

26. Dong H, Zhu G, Tamada K, et al. B7-H1, a third member of the B7 family, co-stimulates T-cell proliferation and interleukin-10 secretion. Nat Med 1999;5:1365-9.

27. He Y, Liu S, Mattei J, et al. The combination of anti-KIR monoclonal antibodies with anti-PD-1/PD-L1 monoclonal antibodies could be a critical breakthrough in overcoming tumor immune escape in NSCLC. Drug Des Devel Ther 2018;12:981-6. 